\title{
UIT Astro-2 Observations of NGC 4449
}

\author{
Robert S. Hill*, Michael N. Fanelli*, Denise A. Smith ${ }^{\dagger}$, Ralph C. \\ Bohlin ${ }^{\ddagger}$, Susan G. Neff", Robert W. O’Connell ${ }^{\mp}$, Morton S. \\ Roberts $^{\S}$, Andrew M. Smith॥, Theodore P. Stecher** \\ ${ }^{*}$ Hughes STX Corp., Code 681, NASA/GSFC, Greenbelt, MD 20771 \\ $\dagger N R C$, Code 681, NASA/GSFC, Greenbelt, MD 20771 \\ ${ }^{\ddagger}$ Space Telescope Science Insitute, 3700 San Martin Drive, Baltimore, MD 21218 \\ "Code 681, NASA/GSFC, Greenbelt, MD 20771 \\ ฯ University of Virginia, P. O. Box 3818, Charlottesville, VA 22903 \\ $\S$ National Radio Astronomy Observatory, Edgemont Road, Charlottesville, VA 22903 \\ ${ }^{* *}$ Code 680, NASA/GSFC, Greenbelt, MD 20771
}

\begin{abstract}
The bright Magellanic irregular galaxy NGC 4449 was observed by the Ultraviolet Imaging Telescope (UIT) during the Astro-2 Spacelab mission in March, 1995. Far ultraviolet (FUV) images at a spatial resolution of $\sim 3$ " show bright starforming knots that are consistent with the general optical morphology of the galaxy and are often coincident with bright H II regions. Comparison of FUV with $\mathrm{H} \alpha$ shows that in a few regions, sequential star formation may have occurred over the last few Myr. The bright star forming complexes in NGC 4449 are superposed on a smooth, diffuse FUV background that may be associated with the $\mathrm{H} \alpha$ "froth."
\end{abstract}

\section{OBSERVATIONS}

NGC 4449 is a bright Magellanic irregular galaxy at a distance of $5.4 \mathrm{Mpc}$ [1]. Its absolute FUV magnitude is estimated at -20.2 , as compared to -18.8 for the Large Magellanic Cloud (LMC), -19.3 for the Local Group Sc spiral M33, and -21.8 for the SBc spiral M83 [2]. In other words, NGC 4449 is comparable to conspicuous, star-forming spirals in FUV luminosity, and it is brighter than the LMC by a factor of $\sim 3.5$. This signature of active, ongoing star formation is confirmed by $\mathrm{H} \alpha$ imagery [3], which shows a large number of compact, bright HII regions against a background of loops, filaments, and diffuse emission. The southern bar is a region of particular complexity, with many bright near-point sources and with bright filamentary structure seen in line emission.

NGC 4449 was observed by the Ultraviolet Imaging Telescope (UIT [4]) during the Astro-2 Space Shuttle mission in March, 1994. UIT observations on the Astro-2 


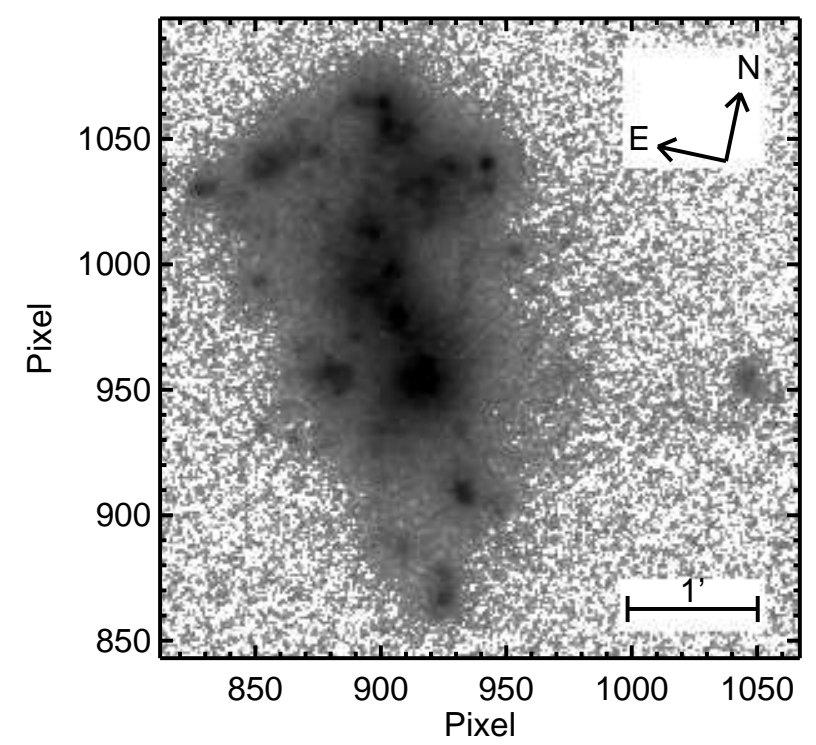

FIGURE 1. UIT FUV Image of NGC 4449 (B1 Filter).

mission were in one of the FUV bands, usually either B1 $(\lambda=152 \mathrm{~nm}, \Delta \lambda=35$ $\mathrm{nm})$ or B5 $(\lambda=162 \mathrm{~nm}, \Delta \lambda=22 \mathrm{~nm})$. The two bandpasses are similar; B5 has a longer short-wavelength cutoff to exclude dayglow.

Observations of NGC 4449 were obtained in both B1 and B5. In this paper, we discuss the $900 \mathrm{~s}$ B1 image, which is the deepest one (Figure 1), and the $500 \mathrm{~s}$ B5 image, which has the best spatial resolution.

NGC 4449 was also observed from the ground using the Goddard Fabry-Perot Imager (GFPI). These observations, made by K.-P. Cheng, R. Oliversen, and P. M. N. Hintzen, are discussed in an earlier paper based on FUV sounding rocket imagery [2]. The GFPI data consist of emission-line images in $\mathrm{H} \alpha, \mathrm{H} \beta$, and nearby, narrow continuum bands.

As a result of analyzing the UIT data, the calibration of observations reported in the sounding rocket paper [2] has been revised. In all bands, the reported fluxes are a factor of $\sim 2$ too high. Specifically, any single Balmer line flux should be multiplied by 0.6 , and any single rocket UV flux should be multiplied by $\sim 0.5$. The off-band visual continuum measurements are affected in the same way as the line fluxes. In the UV case, the correction is less well defined because of a somewhat uncertain bad-pixel factor, varying from source to source, which is included in the rocket data, but is unnecessary for the UIT data. In summary, the effect on any ratio of two fluxes is $\sim 20 \%$. Therefore, the modeled source ages reported in the rocket paper are not significantly affected. 
TABLE 1. Photometry of Selected Froth Regions

\begin{tabular}{cccc}
\hline No. & $\begin{array}{c}F_{H \alpha} \\
\left(\mathrm{erg} \mathrm{cm}^{-2} \mathrm{~s}^{-1}\right)\end{array}$ & $\begin{array}{c}f_{F U V} \\
\left(\mathrm{erg} \mathrm{cm}^{-2} \mathrm{~s}^{-1} \AA^{-1}\right)\end{array}$ & $\begin{array}{c}\text { Equivalent Age } \\
(\mathrm{Myr})\end{array}$ \\
\hline 1 & $2.33 \times 10^{-13}$ & $2.28 \times 10^{-14}$ & 4.0 \\
2 & $1.74 \times 10^{-13}$ & $1.81 \times 10^{-14}$ & 4.2 \\
3 & $1.00 \times 10^{-13}$ & $1.11 \times 10^{-14}$ & 4.4 \\
4 & $1.05 \times 10^{-13}$ & $1.33 \times 10^{-14}$ & 4.8 \\
\hline
\end{tabular}

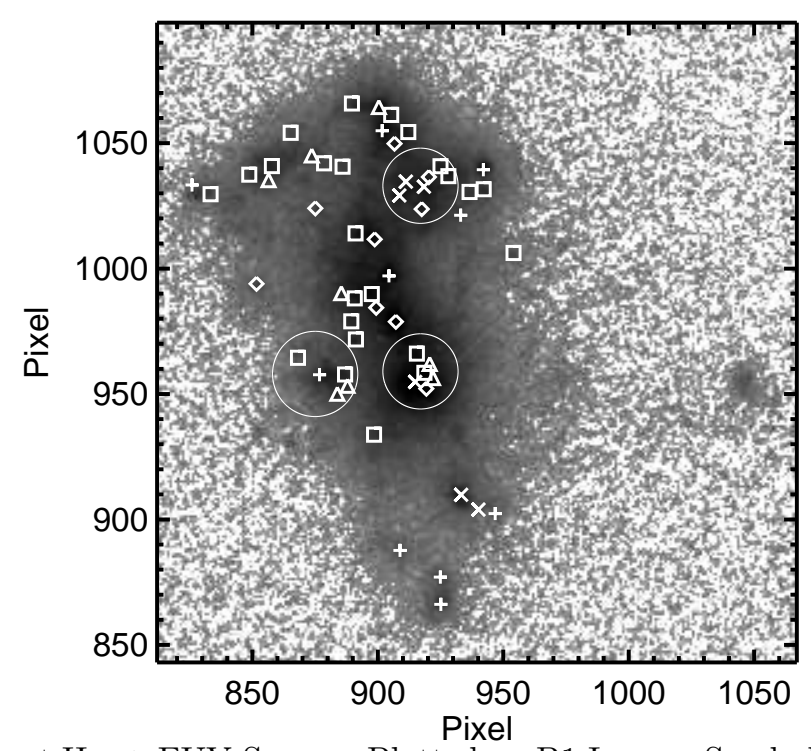

FIGURE 2. Compact $\mathrm{H} \alpha+$ FUV Sources Plotted on B1 Image. Symbols indicate ages in Myr, as follows: triangles, $<1$; squares, $1-3$; diamonds, $3-5$; plusses, $5-7$; crosses, $>7$.

\section{ANALYSIS}

The term froth has been coined to describe a certain morphology of $\mathrm{H} \alpha$-emitting material outside of typical HII regions. Froth lacks conspicuous embedded star clusters, and it is distinguished from simple diffuse emission by a complex structure of bright filaments. The froth is thought to be the product of a mixed ionization mechanism, with energy supplied both by ionizing stars and by shocks [3].

A substantial contribution from photoionization is likely. A plausibility argument for this position can be made with a simple application of the FUV image data. We examine the ratio between $\mathrm{H} \alpha$ surface brightness in any given local area and the coincident FUV surface brightness. If the ratio of $\mathrm{H} \alpha$ to FUV is consistent with that expected from an ionizing cluster, then photoionization is a plausible mechanism. Apertures 20" in radius are superposed on 4 regions of diffuse, filamentary $\mathrm{H} \alpha$ emission and extended UV emission. The $\mathrm{H} \alpha$ and FUV fluxes for these regions are given in Table 1, together with the implied ages [2], without extinction corrections or background flux subtraction. The implied FUV surface brightnesses are equivalent to 1 or 2 unreddened early B stars per $\operatorname{arcsec}^{2}$. 
In the case of compact, bright knots of $\mathrm{H} \alpha$ and FUV emission, the age computed from the ratio of the two fluxes has real meaning as the possible age of a coeval cluster of stars. Ages are computed from $\mathrm{H} \alpha$ to FUV (B5) flux ratios in circular apertures. Sources are grouped by proximity and a background surface brightness within NGC 4449 is subtracted from each group. Extinction is computed using the Balmer decrement, assuming that $A_{F U V} / E(B-V)=4.5$, which is similar to the Orion Nebula extinction curve [5]. Figure 2 shows a map of the compact source ages. Three regions showing a spatial progression of ages are circled. These patterns may indicate sequential star formation of the type discussed by Elmegreen \& Lada $[6,7]$. In this scenario, the typical spatial separation between stellar generations appears to be $\sim 100-300 \mathrm{pc}$, a factor of $\sim 5$ larger than in Elmegreen \& Lada's Galactic examples.

\section{REFERENCES}

1. Bomans, D. J., Chu, Y.-H., \& Hopp, U. 1997, AJ, in press

2. Hill, R. S., Home, A. T., Smith A. M., Bruhweiler, F. C., Cheng, K.-P., Hintzen, P. M. N., \& Oliversen, R. J. 1994, ApJ, 430, 568

3. Hunter, D. A. \& Gallagher, J. S. 1900, ApJ, 362, 480

4. Stecher, T. P. et al. 1992, ApJ, 395, L1

5. Bohlin, R. C. \& Savage, B. D. 1981, ApJ, 249, 109

6. Elmegreen, B. G. \& Lada, C. J. 1977, ApJ, 214, 725

7. Home, A. T., private communication 